\title{
High Dynamic Range THz Systems using ErAs:In(Al)GaAs Photoconductors
}

\author{
Uttam Nandi, ${ }^{\# 1}$ Fahd R. Faridi, ${ }^{\# 2}$ Anuar D. J. Fernandez Olvera, ${ }^{\# 3}$ Justin Norman, ${ }^{* 4}$ Hong Lu, ${ }^{+5}$ Arthur C. Gossard, ${ }^{* 6}$ \\ Sascha Preu ${ }^{\# 7}$ \\ \#Terahertz Devices and Systems, TU Darmstadt, Darmstadt, Germany \\ *Materials Dept., University of California, Santa Barbara, Santa Barbara, USA \\ ${ }^{+}$College of Engineering and Applied Sciences, University of Nanjing, Nanjing, China \\ $\left\{{ }^{1}\right.$ nandi, ${ }^{2}$ faridi, ${ }^{3}$ fernandez, ${ }^{7}$ preu $\} @$ imp.tu-darmstadt.de, ${ }^{5}$ hlu@ @ ju.edu.cn, ${ }^{4}$ jcnorman223@ gmail.com, \\ ${ }^{6}$ gossard@engineering.ucsb.edu
}

\begin{abstract}
This paper reviews progress on ErAs:In(AI)GaAs photomixers for operation with telecom lasers at $1550 \mathrm{~nm}$, including linearity and absorption coefficient measurements, specifications, packaging example, and applications in vector spectrometry. We have achieved a receiver noise equivalent power as low as $1.81 \mathrm{fW} / \mathrm{Hz}$ at 188 $\mathrm{GHz}$ under continuous-wave operation and a bandwidth of more than $6 \mathrm{THz}$ and a peak dynamic range of $89 \mathrm{~dB}$ under pulsed operation.

Keywords - terahertz, photoconductor, telecom wavelength, continuous-wave, time domain spectroscopy, vector spectroscopy.

(C) 2018 IEEE. Published version at:

https://ieeexplore.ieee.org/abstract/document/8880067
\end{abstract}

\section{INTRODUCTION}

Within the past two decades, room-temperature operated Terahertz (THz, $100 \mathrm{GHz}-10 \mathrm{THz})$ sources and receivers have advanced rapidly, including electronic [1], optical [2] and photonic devices. Photonic systems consist of photoactive semiconductor devices that are driven by a laser signal. The laser signal consists either of a single, 100 fs-scale pulse with a bandwidth of several $\mathrm{THz}$ or of two heterodyned continuous-wave (CW) laser beams with a frequency difference in the $\mathrm{THz}$ range. The semiconductor device absorbs the laser signal, effectively resulting in mixing the frequency components of the optical signal and consequently generating an $\mathrm{AC}$ current at these frequencies. In most cases, the current is fed into an antenna to emit $\mathrm{THz}$ radiation. Electrical systems yet offer the largest dynamic range in the range of $120 \mathrm{~dB}$ around $300 \mathrm{GHz}$ [1], however, at cost of bandwidth which is usually $50 \%$ of the center frequency at best. Photonic systems have caught up in the past years, yet offering a peak dynamic range around 105 $\mathrm{dB}$ [3] while they cover at the same time a bandwidth of several $\mathrm{THz}$ with a single system. For this reason, many spectroscopic applications have been performed with photonic systems. A further field of applications is communication where the photonic device down-converts a data stream encoded on a $1550 \mathrm{~nm}$ signal to a mm-wave wireless signal [4] or to an electrical signal for a data processing unit. Industrial applications are in the field of non-destructive testing, e.g. [5].
Photoconductors, play an essential role in almost all photonic Terahertz systems. Photoconductors are optically switched resistors with sub-ps recovery time. Upon optical excitation, they become conductive due to generation of electron-hole pairs via absorption. In order to respond to a THz-modulated signal, the carriers are trapped on a sub-ps time scale by specially designed defects therefore removing the carriers from transport and restoring the initial high resistance.

In pulsed systems, photoconductors serve as both sources and receivers. Below $4 \mathrm{THz}$, well designed photoconductors outperform nonlinear crystals that can be alternatively used. In $\mathrm{CW}$ systems, they are frequently employed as homodyne receivers.

Of particular importance nowadays is compatibility with $1550 \mathrm{~nm}$ driving lasers in order to make use of telecom lasers and components for the optical part of the system which is a major cost factor. This imposes certain requirements on the photoconductive material. First, it must be capable to absorb the $1550 \mathrm{~nm}$ laser signal. Most efficient are interband transitions that require a band gap energy below $0.8 \mathrm{eV}$. InGaAs has proven to be a viable material for this purpose. Unfortunately, the low band gap, $E_{G}$, reduces the maximum bias field, $E_{b d}$, that can be applied to the device which empirically scales as $E b d \sim E G^{2.5}$ [6] and increases undesired dark currents. These limitations reduce the emitted $\mathrm{THz}$ power of photoconductive sources as the $\mathrm{THz}$-modulated resistance must be DC biased in order to generate a $\mathrm{THz}$ current. Further requirements are a large carrier mobility in order to maximize the generated current at a given DC bias and, in the case of CW devices and receivers in general, a short carrier lifetime which allows the material to recover to the high resistance after optical excitation.

This paper summarizes recent results of telecomwavelength compatible ErAs:In(Al)GaAs photoconductors with outstanding performance parameters.

\section{THE MATERIAL SYSTEM}

ErAs:(InAl)GaAs photoconductors consist of a superlattice structure of semi-metallic ErAs clusters that act 
as efficient carrier trapping centers and an InGaAs absorber. For sources, InAlAs layers are further included. Typical band structures for sources and receivers are illustrated in Fig. 1 a) and b) respectively. The devices typically consist of 70-100 superlattice periods of the illustrated structures. Further details can be found in [7] and [8]. For both sources and receivers, the absorption takes place in the InGaAs layers. Carriers then drift to the ErAs clusters where they are trapped. For sources, they need to tunnel through adjacent $\mathrm{p}$ doped InAlAs layers with a thickness in the range of $2 \mathrm{~nm}$. These layers assist to improve the device resistance.

Fig. 2 shows the absorption profile of a receiver structure with a thickness of the active layer of $1 \mu \mathrm{m}$. The absorption coefficient is around $5500-7100 / \mathrm{cm}$ for the telecom wavelength range (1525-1600 $\mathrm{nm})$. The carrier lifetime of the receivers is in the range of 510fs [9], while Al-containing devices feature lifetimes above 1 ps typically. a)

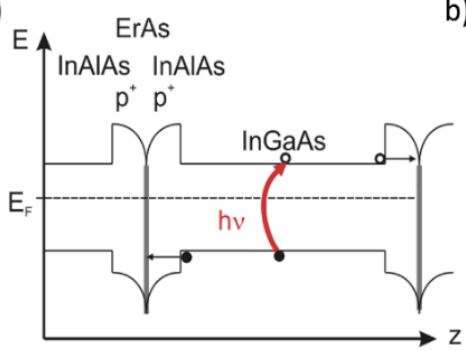

b)

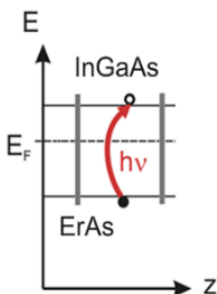

Fig. 1. a) Typical band structure of an ErAs:In(Al)GaAs source and (b) an ErAs:InGaAs receiver.

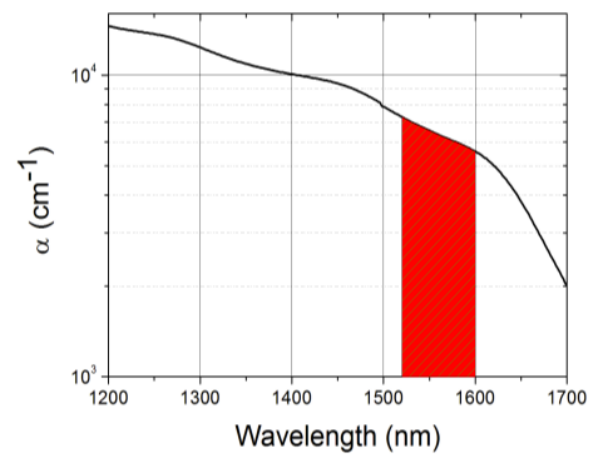

Fig. 2. Spectrally resolved absorption coefficient of an ErAs:InGaAs receiver

\section{RESULTS}

Inset of Fig. 3 illustrates a packaging example of an ErAs: $\operatorname{In}(\mathrm{Al}) \mathrm{GaAs}$ photoconductor that is frequently used in experiments. Under $\mathrm{CW}$ operation, the receivers feature a measured noise equivalent power (NEP) as low as 1.81 $\mathrm{fW} / \mathrm{Hz}$ at $188 \mathrm{GHz}$ [10] which increases to about $100 \mathrm{fW} / \mathrm{Hz}$ at $1 \mathrm{THz}$. These data are as recorded and not idealized values. The intrinsic, theoretical NEP limit at $200 \mathrm{GHz}$ is estimated to be in the low aW/Hz range, given perfect $\mathrm{THz}$ coupling and negligible external noise contributions [10]. The devices feature a large linearity range. At the maximum available power of a CW system of $150 \mu \mathrm{W}$ at $200 \mathrm{GHz}$ we did not see any saturation. We did see some saturation for $\mathrm{THz}$ powers beyond the several $\mathrm{mW}$ level using a backward wave oscillator at $100 \mathrm{GHz}$, however, the devices tend to take damage at these high power levels. With an ErAs: In(Al)GaAs source, we achieved a dynamic range of $78 \mathrm{~dB}$ at an integration time of $300 \mathrm{~ms}$ with a laser power of only $26 \mathrm{~mW}$ in each photoconductor, i.e. source and receiver [7]. The extrapolated bandwidth is about 3.6 THz. Combined with powerful $\mathrm{p}-\mathrm{i}-\mathrm{n}$ diode-based photomixer sources, peak dynamic ranges beyond $100 \mathrm{~dB}$ should be feasible.

Under pulsed operation, ErAs: $\operatorname{In}(\mathrm{Al}) \mathrm{GaAs}$ devices are both excellent sources and receivers. Fig. 3 depicts a measurement with almost $90 \mathrm{~dB}$ peak dynamic range and a bandwidth beyond $6.2 \mathrm{THz}$. The source was composed of 90 periods of $15 \mathrm{~nm}$ InGaAs, $1.5 \mathrm{~nm}$ InAlAs, $0.8 \mathrm{ML}$ p-deltadoped ErAs, and $1.5 \mathrm{~nm}$ InAlAs (Fig. 1a), attached to a 25 $\mu \mathrm{m}$ slotline antenna. The receiver consisted of 100 periods of $10 \mathrm{~nm}$ InGaAs, and 0.8 ML p-delta-doped ErAs (Fig.1 b) equipped with a $25 \mu \mathrm{m}$ H-dipole antenna with a gap of $5 \mu \mathrm{m}$. The measurement was taken at a source bias of $150 \mathrm{~V}$, a source laser power of $45 \mathrm{~mW}$ and a receiver laser power of $16 \mathrm{~mW}$ generated by a fiber-coupled Menlo C-fiber system. At this laser power, the source can be operated with $200 \mathrm{~V}$ bias without taking damage, i.e. the presented spectrum is obtained under safe operation conditions.

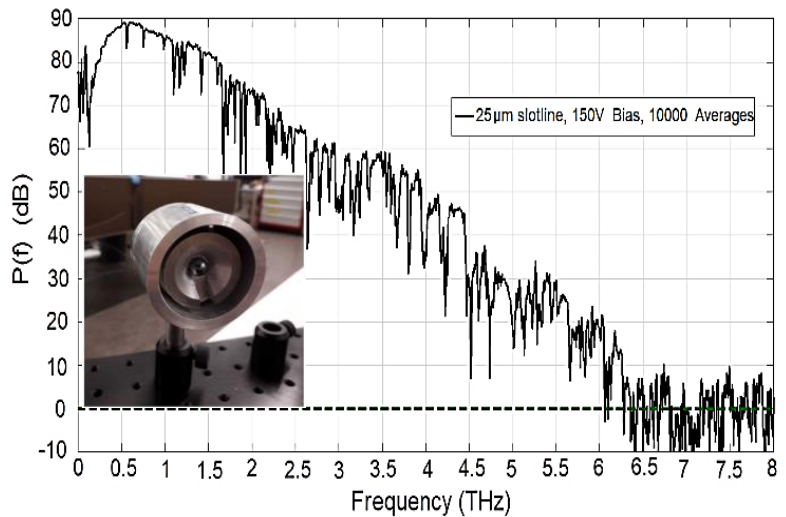

Fig. 3. Spectrum obtained with only ErAs: $\operatorname{In}(\mathrm{Al}) \mathrm{GaAs}$ photoconductors with fiber-coupled $1550 \mathrm{~nm}$ pulses as described in the text. The measurement time was approximately 17 minutes. Inset: APC connectorized packaging example including a $1 \mathrm{~cm}$ diameter silicon lens.

\section{APPLICATION EXAMPLE: 1.5 PORT VECTOR SPECTROMETER}

This section summarizes recent results of a pulsed 1.5 port Terahertz time domain (TDS) vector spectrometer as discussed in ref. [11], using only ErAs: $\operatorname{In}(\mathrm{Al}) \mathrm{GaAs}$ photoconductors. The setup is illustrated in Fig. 4. The system uses three laser signals that originate from the same oscillator. One signal drives an $\operatorname{ErAs}: \operatorname{In}(\mathrm{Al}) \mathrm{GaAs}$ source (Tx), the other two laser signals are guided through a delay stage each and drive two receivers (Rx1, Rx2). Rx1 measures the transmitted $\mathrm{THz}$ signal through a sample under test, whereas Rx2 measures the reflected signal. The wire grid polarizers (WGP) are implemented as beam splitters. WGP1 is aligned to $45^{\circ}(\sim 3 \mathrm{~dB}$ loss $)$ with respect to the source polarization while WGP2 is vertical. This way, the reflected signal from the sample is again reflected by WGP1 towards $\mathrm{Rx} 2$ with an additional $3 \mathrm{~dB}$ loss. The setup is optimized for measuring the refractive index, the absorption coefficient and the thickness of a plane sample by a $\mathrm{THz}$ TDS. There is no mechanical measurement of the sample thickness necessary. Fig. 5 illustrates the measurement principle at the example of a high resistivity silicon wafer with a nominal thickness of $500 \mu \mathrm{m}$ (manufacturer specification). 
The transmission trace (under ambient air) shows a time delay between the pulse recorded with the empty setup (red) and the sample introduced in the setup (blue). The temporal distance between the two main peaks is $\Delta t_{1}=(n-1) d / c 0$, where $c_{0}$ is the vacuum speed of light, $n$ is the refractive index of the material under test and $d$ is the mechanical thickness. For simplicity, we assume the refractive index of air to be 1 . The distance between the main peak and the first echo in both the transmitted and reflected signal is $\Delta t_{2}=$ $2 n d / c_{0}$. Usually the reflection measurement is better suited for lossy samples than the transmission measurement as the echo in the transmission measurement is often hardly visible. Therefore, the data quality is better for a synchronous measurement of $R$ and $T$, resulting in reduced errors and larger bandwidth [11]. The equation system for $\Delta t_{1}$ and $\Delta t 2$ allows to solve for $n$ and $d$ individually. The absorption coefficient can be extracted from the transmission measurement amplitude. As the refractive index and the absorption coefficient may be frequency-dependent, the equation system is numerically evaluated by an algorithm for the frequency range under investigation [11]. For the silicon wafer, we determine a thickness of $498 \pm 10 \mu \mathrm{m}$ with a higher accuracy than a caliper measurement and in excellent agreement with the thickness of $500 \mu \mathrm{m}$ according to the manufacturer specification. The refractive index is frequency-independent with a value of $3.25 \pm 0.01$ which is about $5 \%$ smaller than the literature value of 3.416. The absorption for the high resistivity silicon wafer is below the resolution limit. Though the presented characterization of a silicon wafer is a simple example, the setup and the algorithm is capable of characterizing more complex structures, including non-reciprocal devices such as isolators [12].

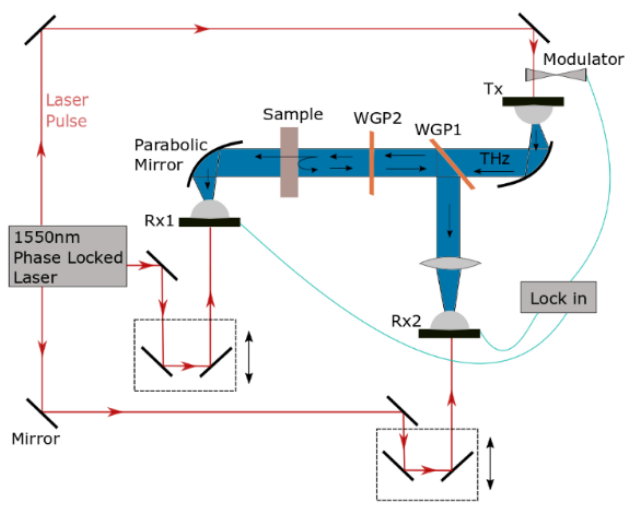

Fig. 4. Schematic of the 1.5 port vector spectrometer setup. Red lines: $1550 \mathrm{~nm}$ laser signals, blue: THz path.

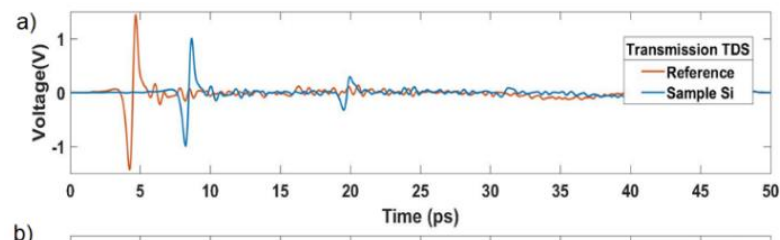

$$
\text { b) }
$$

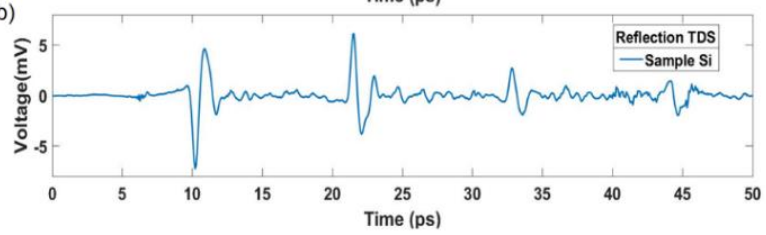

Fig. 5. Time domain traces for the transmission (a) and reflection (b).

\section{CONCLUSION}

ErAs:In(Al)GaAs photoconductors are versatile, powerful devices for both TDS and photonic CW systems at a competitive level with the best devices reported so far $[3,13]$. We have shown a first application of these devices in a 1.5 port vector spectrometer that measures the reflection and transmission synchronously. This improves the data quality and extends frequency coverage as compared to a standard TDS measurement.

\section{ACKNOWLEDGMENT}

We acknowledge the German Research Foundation (DFG), for funding project 278381540 (REPHCON). This project has further received funding from the European Research Council (ERC) under the European Union's Horizon 2020 research and innovation program (GA No. 713780)

\section{REFERENCES}

[1] http://www.vadiodes.com/en/wr3-4vnax, accessed April $23^{\text {rd }} 2019$

[2] S.I. Hayashi, K. Nawata, T. Taira, J.I. Shikata, K. Kawase, and H. Minamide, "Ultrabright continuously tunable terahertz-wave generation at room temperature," Scientific reports 4, p. 5045, 2014.

[3] T. Göbel, D. Stanze, B. Globisch, R. J. B. Dietz, H. Roehle, and M. Schell, "Telecom technology based continuous wave terahertz photomixing system with 105 decibel signal-to-noise ratio and 3.5 terahertz bandwidth," Optics Lett., vol. 38, pp. 4197-4120, Oct. 2013

[4] M. Freire Hermelo, P.-T. Shih, M. Steeg, A. Ng'oma, and A. Stöhr, "Spectral efficient 64-QAM-OFDM terahertz communication link," Opt. Express, vol. 25, pp. 19360-19370, 2017.

[5] M. Stecher, C. Jördens, N. Krumbholz, C. Jansen, M. Scheller, R. Wilk, O. Peters, B. Scherger, B. Ewers, and M. Koch, "Towards Industrial Inspection with THz Systems" in Ultrashort pulse laser technology Springer International Publishing, pp. 311-335, 2016.

[6] J. L. Hudgins, G. S. Simin, E. Santi, and M. A. Khan, "An assessment of wide bandgap semiconductors for power devices," IEEE Trans. Power Electron., vol. 18, pp. 907-914, 2003.

[7] A.d.J. Fernandez Olvera, H. Lu, A. Gossard, and S. Preu, "Continuous-wave $1550 \mathrm{~nm}$ operated terahertz system using ErAs: $\operatorname{In}(\mathrm{Al}) \mathrm{GaAs}$ photo-conductors with $52 \mathrm{~dB}$ dynamic range at 1 THz," Opt. Express, vol. 25, pp. 29492-29500, 2017.

[8] U. Nandi, et al. "1550-nm Driven ErAs: In (Al) GaAs Photoconductor-Based Terahertz Time Domain System with $6.5 \mathrm{THz}$ Bandwidth." Journal of Infrared, Millimeter, and Terahertz Waves, vol. 39, pp. 340-348, 2018.

[9] J. Suen, P. Krogen, S. Preu, H. Lu, A. Gossard, D. Driscoll, and P. Lubin, "Measurement and modeling of ErAs:In0.53Ga0.47As nanocomposite photoconductivity for $\mathrm{THz}$ generation at $1.55 \mu \mathrm{m}$ pump wavelength," J.of Appl. Phys., vol. 116, p. 013703, 2014.

[10] A.d.J. Fernandez Olvera, A. Roggenbuck, K. Dutzi, N. Vieweg, H. Lu, A. C. Gossard and S. Preu, "International System of Units (SI) Noise-Equivalent Power and Responsivity Characterization of Continuous Wave ErAs:InGaAs Photoconductive Terahertz Detectors," Photonics, vol. 6, pp. 15 (2019).

[11] F. R. Faridi, U. Nandi, and S. Preu, "1.5 Port Vector Spectrometer for Terahertz Time Domain Spectroscopy," in 2018 43rd International Conference on Infrared, Millimeter, and Terahertz Waves (IRMMWTHz), pp. 1-2, 2018

[12] F.R. Faridi and S. Preu "Characterization of a Terahertz Isolator using a 1.5 port Vector Spectrometer" submitted to the $44^{\text {th }}$ International Conference on Infrared, Millimeter, and Terahertz Waves (IRMMW-THz), 2019.

[13] M. Yahyapour, A. Jahn, K. Dutzi, T. Puppe, P. Leisching, B. Schmauss, N. Vieweg, and A. Deninger, "Fastest Thickness Measurements with a Terahertz Time-Domain System Based on Electronically Controlled Optical Sampling," Appl. Sciences, vol. 9, 1283,2019 
(C) 2018 IEEE. Personal use of this material is permitted. Permission from IEEE must be obtained for all other uses, in any current or future media, including reprinting/ republishing this material for advertising or promotional purposes, creating new collective works, for resale or redistribution to servers or lists, or reuse of any copyrighted component of this work in other works.
Published article: Uttam Nandi, Fahd R. Faridi, Anuar D. J. Fernandez Olvera, Justin Norman, Hong Lu, Arthur C. Gossard, Sascha Preu, High Dynamic Range THz Systems using ErAs:In(Al)GaAs Photoconductors. In: 2019 IEEE MTT-S International Microwave Workshop Series on Advanced Materials and Processes for RF and $\mathrm{THz}$ Applications (IMWSAMP). IEEE, 2019. S. 115-117.

DOI: $\underline{\text { 10.1109/IMWS-AMP.2019.8880067 }}$ 